Appl. Set-Valued Anal. Optim. 3 (2021), No. 2, pp. 221-237

Available online at http://asvao.biemdas.com

https://doi.org/10.23952/asvao.3.2021.2.07

\title{
THE EXISTENCE AND APPROXIMATION FOR SOLUTIONS OF VARIATIONAL INCLUSION PROBLEMS
}

\author{
E.U. OFOEDU*, K.O. IBEH, C.B. OSIGWE, L.O. MADU, C.G. EZEA \\ Department of Mathematics, Nnamdi Azikiwe University, Awka, Nigeria
}

\begin{abstract}
In this paper, we investigate the existence of solutions of a variational inclusion problem. An iterative algorithm is investigated with the aid of Michael's selection theorem and Nadler's theorem. A strong convergence theorem is established in the framework of real Banach spaces.
\end{abstract}

Keywords. Variational inclusion; Variational inequality; $m$-accretive mapping; Nonexpansive mapping.

\section{INTRODUCTION}

In the current research trends, nonlinear optimization problems with set-valued operators have attracted much attention since they find a number of real applications in the real world, such as, machine learning, medical imaging, signal processing, traffic and transportation; see, e.g., $[1,2,3,4]$ and the references therein.

Let $H$ be a real Hilbert space, and let $C(H)$ denote the family of all nonempty compact subsets of $H$. Let $A$ be a mapping on $H$. Throughout this paper, we denote the domain and range of $A$ by $D(A)$ and $R(A)$, respectively.

Recall the following set-valued variational inclusion problems in $H$. For a given maximal monotone mapping $A: H \rightarrow H$, a nonlinear mapping $N: H \times H \rightarrow H$, set-valued mappings $T, F: H \rightarrow C(H)$, and a single-valued mapping $g: H \rightarrow H$, a fixed $f \in H$, find $u \in H, w \in T(u)$, and $y \in F(u)$ such that

$$
f \in N(w, y)+A(g(u))
$$

If $f=0$, it is reduced to the set-valued variational inclusion studied by Noor [5].

Using the resolvent operator technique, some existence theorems and convergence theorems were established for the variations of set-valued variational inclusion (1.1) recently; see, e.g., $[5,6,7,8,9,10,11,12,13,14,15,16,17,18,19,20]$ and the references therein.

${ }^{*}$ Corresponding author.

E-mail addresses: eu.ofoedu@unizik.edu.ng, euofoedu@yahoo.com (E.U. Ofoedu), ibehkingsleyobinna@gma il.com (K.O. Ibeh); chimezieosigwe@ gmail.com (C.B. Osigwe); m.ogechukwu@ yahoo.com (L.O. Madu); chineduezea@yahoo.com (C.G. Ezea).

Received March 4, 2020; Accepted May 15, 2020.

(C)2021 Applied Set-Valued Analysis and Optimization 
Let $C B(E)$ be the family of all nonempty closed and bounded subsets of $E$. The Hausdorff metric $\Theta$ on $C B(E)$ is defined by

$$
\Theta(A, B)=\max \left\{\sup _{x \in A} d(x, B), \sup _{y \in B} d(A, y)\right\}, A, B \in C B(E) .
$$

A set-valued mapping $T: E \rightarrow C B(E)$ is said to be a $\phi$-contractive mapping (with respect to the Hausdoff metric) if there exists a s continuous increasing function $\phi:[0,+\infty) \rightarrow[0,+\infty)$ with $\phi(0)=0$ such that, for any $x, y \in D(T)$,

$$
\Theta(T x, T y) \leq \phi(\|x-y\|) .
$$

The mapping $T$ is called a Lipschitz continuous mapping (with respect to the Hausdorff metric) if there exists a constant $L>0$ such that $x, y \in D(T)$,

$$
\Theta(T x, T y) \leq L\|x-y\| \text {. }
$$

It is easy to see that every Lipschitz continuous set-valued mapping is $\phi$-contractive with respect to the function $\phi:[0,+\infty) \rightarrow[0,+\infty)$ given for all $t \in[0,+\infty)$ by $\phi(t)=L t$.

If $T: E \rightarrow E$ is single-valued mapping, then $T$ is $\phi$-contractive if there exists a continuous increasing function $\phi:[0,+\infty) \rightarrow[0,+\infty)$ with $\phi(0)=0$ such that, for any $x, y \in D(T)$,

$$
\|T x-T y\| \leq \phi(\|x-y\|) \text {. }
$$

The single-valued mapping $T$ is called a Lipschitz continuous mapping if there exists a constant $L>0$ such that $x, y \in D(T)$,

$$
\|T x-T y\| \leq L\|x-y\| \text {. }
$$

It is well known that every single-valued Lipschitz mapping is uniformly continuous (where a mapping $T: E \rightarrow E$ is uniformly continuous if, for all $\varepsilon>0$, there exists a $\delta(\varepsilon)>0$ such that, for all $x, y \in D(T)$ satisfying $\|x-y\|<\delta(\varepsilon),\|T x-T y\| \leq \varepsilon$ ). It is also known (see, e.g., Alber et al. [21]) that if $T: E \rightarrow E$ is a uniformly continuous mapping, then there exists a continuous increasing mapping $\pi: \mathbb{R} \rightarrow \mathbb{R}$ with $\pi(0)=0$ (which is called the modulus of uniform continuity) such that, for any $x, y \in D(T),\|T x-T y\| \leq \pi(\|x-y\|)$. Thus, in the singlevalued case, the class of $\phi$-contractive mappings includes the class of uniformly continuous mappings. So, the typical example of class of $\phi$-contractive single-valued mappings is the class of uniformly continuous mappings which is a proper supper class of single-valued Lipschitz mappings. In view of this, it is natural to extend the definition of $\phi$-contractive mappings to the set-valued case.

In 2002, Chang, Kim, and Kim [6] obtained the following result.

Theorem 1.1. Let $E$ be a real uniformly smooth Banach space. Let $T, F: E \times E \rightarrow C B(E), A$ : $D(A) \subseteq E \rightarrow 2^{E}$ be three set-valued mappings. Let $N: E \times E \rightarrow E$ be a single-valued continuous mapping, and let $g: D(A) \rightarrow D(A)$ be a single-valued continuous mapping satisfying the following conditions,

(i) $A \circ g: E \rightarrow 2^{E}$ is m-accretive;

(ii) $T: E \rightarrow C B(E)$ is $\mu$-Lipschitzian continuous;

(iii) $F: E \rightarrow C B(E)$ is $\xi$-Lipschitzian continuous, where $\mu$ and $\xi$ are positive constants;

(iv) the mapping $x \mapsto N(x, y)$ is $\varphi$-strongly accretive with respect to the mapping $T$;

(v) the mapping $y \mapsto N(x, y)$ is accretive with respect to the set-valued mapping $F$. 
Then, for any given $f \in E, \lambda>0$, there exists $q \in D(A), w \in T(q), k \in F(q)$ such that $(q, w, k)$ is a solution of the set-valued variational inclusion (1.1). Furthermore, let $D(A)$ is a closed set in $E$, and suppose that $\left\{\alpha_{n}\right\},\left\{\beta_{n}\right\}$ are two sequences in [0,1] satisfying (i) $\lim _{n \rightarrow \infty} \alpha_{n}=0$, $\lim _{n \rightarrow \infty} \beta_{n}=0$, (ii) $\sum_{n=0}^{\infty} \alpha_{n}=\infty$, (iii) $\sum_{n=0}^{\infty}\left\|u_{n}\right\|<\infty$, and $\lim _{n \rightarrow \infty}\left\|v_{n}\right\|=0$. If the ranges $R(I-N(T(),. F())$.$) and R(A \circ g)$ are both bounded and there exists a nonexpansive retraction $Q$ of $E$ onto $D(A)$, then, for any given $x_{0} \in D(A), h_{0} \in T\left(x_{0}\right), z_{0} \in F\left(x_{0}\right)$, the iterative sequences $\left\{x_{n}\right\},\left\{\omega_{n}\right\},\left\{k_{n}\right\},\left\{v_{n}\right\},\left\{h_{n}\right\},\left\{z_{n}\right\}$ and $\left\{p_{n}\right\}$ defined by

$$
\begin{aligned}
x_{n+1} & =Q p_{n}, \\
p_{n} & \in\left(1-\alpha_{n}\right) x_{n}+\alpha_{n}\left(f+Q y_{n}-N\left(\omega_{n}, k_{n}\right)-\lambda A\left(g\left(Q y_{n}\right)\right)\right)+u_{n}, \\
y_{n} & \in\left(1-\beta_{n}\right) x_{n}+\beta_{n}\left(f+x_{n}-N\left(h_{n}, z_{n}\right)-\lambda A\left(g\left(x_{n}\right)\right)\right)+v_{n}, \\
h_{n} & \in T\left(x_{n}\right):\left\|h_{n}-h_{n+1}\right\| \leq\left(1+\frac{1}{n+1}\right) \Theta\left(T x_{n}, T x_{n+1}\right), \\
z_{n} & \in F\left(x_{n}\right):\left\|z_{n}-z_{n+1}\right\| \leq\left(1+\frac{1}{n+1}\right) \Theta\left(F x_{n}, F x_{n+1}\right), \\
\omega_{n} & \in T\left(Q y_{n}\right):\left\|\omega_{n}-\omega_{n+1}\right\| \leq\left(1+\frac{1}{n+1}\right) \Theta\left((Q T y)_{n}, T\left(Q y_{n+1}\right)\right), \\
k_{n} & \in F\left(Q y_{n}\right):\left\|k_{n}-k_{n+1}\right\| \leq\left(1+\frac{1}{n+1}\right) \Theta\left(F\left(Q y_{n}\right), F\left(Q y_{n+1}\right)\right),
\end{aligned}
$$

$n=0,1,2,3, \ldots$, converge strongly to the solution $x, \omega, k$ of the set-valued variational inclusion.

From Theorem 1.1, we have the following observations:

(1) the conditions that the set-valued operators $T: E \rightarrow C B(E)$, and $F: E \rightarrow C B(E)$ are Lipschitz continuous can be extended to the more general case of $\phi$-contractive operators;

(2) the assumption that the operator $x \mapsto N(x, y)$ is $\phi$-strongly accretive with respect to the mapping $T$ can be extended to the more general case of the mapping $x \mapsto N(x, y)$ being $\psi$-uniformly accretive with respect to mapping $T$;

(3) the assumptions on the errors that $\sum_{n=1}^{\infty}\left\|u_{n}\right\|<\infty$ and $\lim _{n \rightarrow \infty}\left\|v_{n}\right\|=0$ are too strong. Borrowing the words of Ofoedu [22], these conditions lead to the definitions of Liu [23]. The most reasonable assumptions on the errors is the one placed by $\mathrm{Xu}$ [24], in which the imposition of the boundedness condition on the errors was made.

\section{PRELIMINARIES}

Throughout this paper, we assume that $E$ is a real Banach space, $E^{*}$ is a topological dual space of $E,\langle.,$.$\rangle is the duality pairing between E$ and $E^{*}$, and $J: E \rightarrow 2^{E^{*}}$ is the normalized duality mapping defined by

$$
J(x)=\left\{f \in E^{*}:\langle x, f\rangle=\|x\| \cdot\|f\|,\|f\|=\|x\|\right\}, \quad \forall x \in E .
$$

Let $A: D(A) \subset E \rightarrow 2^{E}$ be a set-valued mapping. $A$ is said to be accretive if, for any $x, y \in$ $D(A)$, there exists $j(x-y) \in J(x-y)$ such that

$$
\langle u-v, j(x-y)\rangle \geq 0
$$


for all $u \in A x, v \in A y$. $A$ is said to be $\varphi$-strongly accretive if there exists a strictly increasing continuous function $\varphi:[0, \infty) \rightarrow[0, \infty)$ with $\varphi(0)=0$ such that, for any $x, y \in D(A), j(x-y) \in$ $J(x-y)$, and for any $u \in A x, v \in A y$,

$$
\langle u-v, j(x-y)\rangle \geq \varphi(\|x-y\|)\|x-y\| .
$$

$A$ is said to be $\Psi$-uniformly accretive if there exists a strictly increasing continuous function $\Psi:[0, \infty) \rightarrow[0, \infty)$ with $\Psi(0)=0$ such that, for any $x, y \in D(A), j(x-y) \in J(x-y)$ and for any $u \in A x, v \in A y$,

$$
\langle u-v, j(x-y)\rangle \geq \Psi(\|x-y\|) .
$$

$A$ is said to be $m$-accretive if $A$ is accretive and $R(I+\rho A)=E$ for all $\rho>0$, where $I$ is the identity mapping on $E$. $A$ is said to be $\phi$-expansive if, for any $x, y \in D(A)$ and for any $u \in$ $A x, v \in A y$,

$$
\|u-v\| \geq \phi(\|x-y\|) .
$$

It is easy to see that if $A$ is $\varphi$-strongly accretive, then $A$ is $\varphi$-expansive, and every $\varphi$-strongly accretive operator is $\Psi$-uniformly accretive with $\Psi(t)=t \varphi(t), \forall t \in[0,+\infty)$.

Let $T, F: E \rightarrow C B(E)$ be two set-valued mappings. Let $A: E \rightarrow 2^{E}$ be an $m$-accretive mapping, $g: E \rightarrow E$ be a single-valued mapping, and let $N: E \times E \rightarrow E$ be a nonlinear mapping. For any $f \in E, \lambda>0$, we consider the following problem, which consists of finding $q \in E, w \in T(q)$, and $k \in F(q)$ such that

$$
f \in N(w, k)+\lambda A(g(q)) .
$$

This problem is called the set-valued variational inclusion problem in Banach spaces. A number of problems arising in pure and applied sciences can be reduced to the study of this kind of variational inclusion problem (see, e.g., $[5,25,26]$ ).

Remark 2.1. As enumerated in [6], we reconsider and throw more light on some special cases of problem (2.1)

(1) Observe that (1.1) is a special case of (2.1) in the sense that if $E=H$ is a Hilbert space, $A: D(A)=H \rightarrow H$ is a maximal monotone mapping, $T, F: H \rightarrow C(H)$ are two setvalued mappings, and $g: H \rightarrow H$ is a single-valued mapping and $\lambda=1$. Problem (2.1) is equivalent to the problem of finding $q \in H, w \in T(q)$, and $k \in F(q)$ such that

$$
f \in N(w, k)+A(g(q)) .
$$

Problem (1.1) is the set-valued variational inclusion problem, which was introduced and studied by Noor [5] and Noor et al. [11] under some additional conditions.

(2) If $g=I, F=0, T=I, S: E \rightarrow E$ is a single-valued mapping, and $N(x, y)=S x$ for all $(x, y) \in E \times E$, then problem (2.1) is equivalent to the problem of finding $q \in D(A)$ such that $f \in S q+\lambda A q$. This problem was introduced and studied by Jung and Morales [17] and Bruck and Reich [27].

(3) If $E=H$, a real Hilbert space, $\lambda=1$, and $A=\partial \phi$, the subdifferential of a proper convex lower semi-continuous function $\phi: H \rightarrow \mathbb{R} \cup\{+\infty\}$, then problem (2.1) is equivalent to the problem of finding $q \in H, w \in T(q)$, and $k \in F(q)$ such that

$$
\langle N(w, k)-f, x-g(q)\rangle \geq \phi(g(q))-\phi(x), \quad \forall x \in H .
$$


This problem is called the generalized set-valued mixed variational inequality, which was introduced and studied by Noor et al. [11]. Some special cases were also considered in Noor [12]. This problem with $N$ being some special cases was also considered in the setting of Banach spaces (see Chang [7]). Observe that if $g=I, F=0, T=I$, and $S: D(S) \subseteq H \rightarrow H$ a single-valued mapping, $N(x, y)=S(x)$ for all $x \in D(S), y \in H$, and $\phi=0$, then (2.2) is reduced to the problem of finding $q \in D(S)$ such that

$$
\langle S(q), x-q\rangle \geq 0, \quad \forall x \in H .
$$

Problem (2.3) is the classical variational inequality problem studied by many authors under different conditions on operator $S$.

(4) If $E=H$ is a Hilbert space, $T, F, M: H \rightarrow 2^{H}$ are three set-valued mappings, and $m, S, G$ : $H \rightarrow H$ are three single-valued mappings, then $\Gamma_{K}(z)=m(z)+K$, where $K$ is a closed convex subset of $H, N(x, y)=S x+G y$, and

$$
\phi(x)=I_{\Gamma_{K}(z)}(x)= \begin{cases}0, & x \in \Gamma_{K}(z), \\ +\infty, & x \notin \Gamma_{K}(z) .\end{cases}
$$

Then, problem (2.1) is equivalent to the problem of finding $q \in H, w \in T(q)$, and $k \in$ $F(q)$, and $z \in M(q)$ such that

$$
g(q) \in K(q),\langle S w+G k-f, x-g(q)\rangle \geq 0, \quad x \in K(z) .
$$

This problem is called the generalized strongly nonlinear implicit quasi-variational inequality studied by Huang [16].

Consequently (borrowing the summary given in [6]), it follows that for a suitable choice of mappings $T, F, A, g, N$ and space $E$, one can obtain a number of known and new classes of variational inequalities, variational inclusions, and the corresponding optimization problems from set-valued variational inclusion problem (2.1). Furthermore, these types of variational inclusions enable us to study many important problems arising in the mathematical, physical, and engineering sciences in a general and unified framework.

Let $T, F: E \rightarrow 2^{E}$ be two set-valued mappings, and let $N: E \times E \rightarrow E$ be a nonlinear mapping. Recall that the mapping $x \mapsto N(x, y)$ is said to be $\Psi$-uniformly accretive with respect to the mapping $T$ if there exists a strictly increasing continuous function $\Psi:[0, \infty) \rightarrow[0, \infty)$ with $\Psi(0)=0$ such that, for any $x_{1}, x_{2} \in E, j\left(x_{1}-x_{2}\right) \in J\left(x_{1}-x_{2}\right)$, and for any $u_{1} \in T x_{1}, u_{2} \in T x_{2}$, $y \in E$,

$$
\left\langle N\left(u_{1}, y\right)-N\left(u_{2}, y\right), j\left(x_{1}-x_{2}\right)\right\rangle \geq \Psi\left(\left\|x_{1}-x_{2}\right\|\right) .
$$

The mapping $y \mapsto N(x, y)$ is said to be $\Psi$-uniformly accretive with respect to the mapping $F$, if there exists a strictly increasing continuous function $\Psi:[0, \infty) \rightarrow[0, \infty)$ with $\Psi(0)=0$ such that, for any $y_{1}, y_{2} \in E, j\left(y_{1}-y_{2}\right) \in J\left(y_{1}-y_{2}\right)$, and for any $v_{1} \in T y_{1}, v_{2} \in T y_{2}, x \in E$,

$$
\left\langle N\left(x, v_{1}\right)-N\left(x, v_{2}\right), j\left(y_{1}-y_{2}\right)\right\rangle \geq \Psi\left(\left\|y_{1}-y_{2}\right\|\right) .
$$

The mapping $y \mapsto N(x, y)$ is said to be accretive with respect to the mapping $F$, for any $y_{1}, y_{2} \in E, j\left(y_{1}-y_{2}\right) \in J\left(y_{1}-y_{2}\right)$, and for any $v_{1} \in T y_{1}, v_{2} \in T y_{2}, x \in E$,

$$
\left\langle N\left(x, v_{1}\right)-N\left(x, v_{2}\right), j\left(y_{1}-y_{2}\right)\right\rangle \geq 0 .
$$

In the remainder of this paper, the following lemmas play an important role. 
Lemma 2.1. Let $E$ be a real Banach space, and let $J: E \rightarrow 2^{E^{*}}$ be the normalized duality mapping. Then, for any $x, y \in E, j(x+y) \in J(x+y)$, the inequality $\|x+y\|^{2} \leq\|x\|^{2}+2\langle y, j(x+y)\rangle$, holds.

Lemma 2.2. Let $E$ be a real smooth Banach space. Let $T, F: E \rightarrow 2^{E}$ be two set-valued mappings, and let $N: E \times E \rightarrow E$ be a nonlinear mapping satisfying the following conditions: (1) the mapping $x \mapsto N(x, y)$ is $\Psi$-uniformly accretive with respect to the mapping $T$;

(2) the mapping $y \mapsto N(x, y)$ is accretive with respect to the mapping $F$.

Then, the mapping $S: E \rightarrow 2^{E}$, defined by $S x=N(T x, F x), \forall x \in E$, is $\Psi$-uniformly accretive.

Proof. Since $E$ is smooth, one sees that the normalized duality mapping $J: E \rightarrow 2^{E^{*}}$ is singlevalued. So, for any $x_{1}, x_{2} \in E$ and $u_{i} \in S x_{i}, i=1,2$, there exist $w_{i} \in T x_{i}$ and $v_{i} \in F x_{i}$ such that $u_{i}=N\left(w_{i}, v_{i}\right), i=1,2$. By conditions (1) and (2), we have that

$$
\begin{aligned}
\left\langle u_{1}-u_{2}, J\left(x_{1}-x_{2}\right)\right\rangle= & \left\langle N\left(w_{1}, v_{1}\right)-N\left(w_{2}, v_{2}\right), J\left(x_{1}-x_{2}\right)\right\rangle \\
= & \left\langle N\left(w_{1}, v_{1}\right)-N\left(w_{2}, v_{1}\right), J\left(x_{1}-x_{2}\right)\right\rangle \\
& +\left\langle N\left(w_{2}, v_{1}\right)-N\left(w_{2}, v_{2}\right), J\left(x_{1}-x_{2}\right)\right\rangle \\
\geq & \Psi\left(\left\|x_{1}-x_{2}\right\|\right) .
\end{aligned}
$$

This implies that $S=N(T(),. F()$.$) is \Psi$-uniformly accretive.

Lemma 2.3. (Michael's selection theorem [28]). Let $X$ and $Y$ be two Banach spaces, and let $T: X \rightarrow 2^{Y}$ be a lower semi-continuous mapping with nonempty closed convex values. Then, $T$ admits a continuous selection, i.e., there exists a continuous mapping $h: X \rightarrow Y$ such that $h(x) \in T(x)$ for each $x \in X$.

Lemma 2.4. Let $E$ be a real uniformly smooth Banach space, and let $T: E \rightarrow 2^{E}$ be a lower semi-continuous m-accretive mapping. Then, the following conclusions hold:

(1) $T$ admits a continuous and m-accretive selection.

(2) In addition, if $T$ is also $\Psi$-uniformly accretive, then $T$ admits a continuous, $m$-accretive, and $\Psi$-uniformly accretive selection.

Proof. (1) It is well known that if $E$ is a uniformly smooth Banach space and $T: E \rightarrow 2^{E}$ is an maccretive mapping, then, for each $x \in E, T(x)$ is nonempty closed and convex (see, for example, Deimling [25, p.293]). By Michael's theorem, $T$ admits a continuous selection $h: E \rightarrow E$ such that, for all $x \in E, h(x) \in T(x)$.

Next, we prove that $h: E \rightarrow E$ is $m$-accretive. In fact, since $T: E \rightarrow 2^{E}$ is accretive, for any $x, y \in E$ and $u \in T x, v \in T y$, we have $\langle u-v, J(x-y)\rangle \geq 0$. In particular, letting $u=h(x) \in$ $T x$ and $v=h(y) \in T y$, we have

$$
\langle h(x)-h(y), J(x-y)\rangle \geq 0 .
$$

This implies that $h: E \rightarrow E$ is a continuous accretive mapping. By a well-known result due to Martin [29], we have that $h$ is a continuous m-accretive mapping.

(2) In addition, if $T$ is also $\Psi$-uniformly accretive, then the selection $h: E \rightarrow E$ given in (1) is also $\Psi$-uniformly accretive. In fact, for any $x, y \in E$ and $u \in T x, v \in T y$, we have

$$
\langle u-v, J(x-y)\rangle \geq \Psi(\|x-y\|) .
$$


Letting $u=h(x) \in T x$, and $v=h(y) \in T y$, we have

$$
\langle h(x)-h(y), J(x-y)\rangle \geq \Psi(\|x-y\|) .
$$

This implies that $h$ is $\Psi$-uniformly accretive. This completes the proof of this Lemma.

Lemma 2.5. (Nadler's Theorem [26]). Let $E$ be a complete metric space, and let $T: E \longrightarrow$ $C B(E)$ be a set-valued mapping. Then, for any given $\varepsilon>0$ and given $x, y \in E$ and $u \in T x$, there exists $v \in T y$ such that $d(u, v) \leq(1+\varepsilon) \Theta(T x, T y)$, where $\Theta(.,$.$) is the Hausdorff metric$ on $C B(E)$.

Lemma 2.6. (Kazmi [18]). Let $E$ be a uniformly smooth Banach space, and let $A: D(A) \subseteq$ $E \longrightarrow 2^{E}$ be an $m$-accretive and $\phi$-expansive mapping. Then $A$ is surjective.

We now invoke Michael's selection theorem (Lemma 2.3) and Nadler's theorem (Lemma 2.5) to generate the following algorithm for the solution of set-valued variational inclusion (1.1).

Let $\left\{\alpha_{n}\right\},\left\{\hat{\alpha}_{n}\right\}\left\{\beta_{n}\right\}$ and $\left\{\hat{\beta}_{n}\right\}$ be four sequences in $[0,1]$. Let $f \in E$ be any given point, and let $\lambda>0$ be any given positive number. Let $\left\{u_{n}\right\}$ and $\left\{v_{n}\right\}$ be two bounded sequences in $E$. Let $x_{0} \in E$ be arbitrarily given. Let $h, z: E \rightarrow E$ be continuous mappings such that $h\left(x_{0}\right) \in T x_{0}$ and $z\left(x_{0}\right) \in F x_{0}$. Fix

$$
y_{0} \in \hat{\alpha_{0}} x_{0}+\hat{\beta_{0}}\left(f+x_{0}-N\left(h\left(x_{0}\right), z\left(x_{0}\right)\right)-\lambda A\left(g\left(x_{0}\right)\right)\right)+\hat{\gamma}_{0} v_{0} .
$$

Let $w, k: E \rightarrow E$ be continuous mappings such that $w\left(y_{0}\right) \in T\left(y_{0}\right)$ and $k\left(y_{0}\right) \in F y_{0}$. Take

$$
x_{1} \in \alpha_{0} x_{0}+\beta_{0}\left(f+y_{0}-N\left(w\left(y_{0}\right), k\left(y_{0}\right)\right)-\lambda A\left(g\left(y_{0}\right)\right)\right)+\gamma_{0} u_{0} .
$$

From $h\left(x_{0}\right) \in T x_{0}, z\left(x_{0}\right) \in F x_{0}$ and Naddler's theorem (Lemma 2.5), we have that there exists $h_{0} \in T x_{1}$ and $z_{0} \in F x_{1}$ such that

$$
\begin{aligned}
\left\|h\left(x_{0}\right)-h_{0}\right\| & \leq(1+1) \Theta\left(T x_{0}, T x_{1}\right), \\
\left\|z\left(x_{0}\right)-z_{0}\right\| & \leq(1+1) \Theta\left(F x_{0}, F x_{1}\right) .
\end{aligned}
$$

Also, by Michael's seletion theorem, we have $h\left(x_{1}\right) \in T x_{1}$ and $z\left(x_{1}\right) \in F x_{1}$. Fix

$$
y_{1} \in \hat{\alpha_{1}} x_{1}+\hat{\beta}_{1}\left(f+x_{1}-N\left(h\left(x_{1}\right), z\left(x_{1}\right)\right)-\lambda A\left(g\left(x_{1}\right)\right)\right)+\hat{\gamma}_{1} v_{1} .
$$

Again applying Michael's selection theorem, and letting $w, k: E \rightarrow E$ be continuous mappings such that $w\left(y_{0}\right) \in T\left(y_{0}\right)$ and $k\left(y_{0}\right) \in F\left(y_{0}\right)$, we obtain by Nadler's theorem that there exists $w_{0} \in T\left(y_{1}\right)$ and $k_{0} \in F\left(y_{1}\right)$ such that

$$
\begin{aligned}
\left\|w\left(y_{0}\right)-w_{0}\right\| & \leq(1+1) \Theta\left(T\left(y_{0}\right), T\left(y_{1}\right)\right), \\
\left\|k\left(y_{0}\right)-k_{0}\right\| & \leq(1+1) \Theta\left(F\left(y_{0}\right), F\left(y_{1}\right)\right) .
\end{aligned}
$$

Choose

$$
x_{2} \in \alpha_{1} x_{1}+\beta_{1}\left(f+y_{1}-N\left(w\left(y_{1}\right), k\left(y_{1}\right)\right)-\lambda A\left(g\left(y_{1}\right)\right)\right)+\gamma_{1} u_{1} .
$$

Since $h\left(x_{1}\right) \in T x_{1}, z\left(x_{1}\right) \in F x_{1}$, then by Naddler's theorem there exists $h_{1} \in T x_{2}$ and $z_{1} \in F x_{2}$ such that

$$
\begin{aligned}
\left\|h\left(x_{1}\right)-h_{1}\right\| & \leq\left(1+\frac{1}{2}\right) \Theta\left(T x_{1}, T x_{2}\right), \\
\left\|z\left(x_{1}\right)-z_{1}\right\| & \leq\left(1+\frac{1}{2}\right) \Theta\left(F x_{1}, F x_{2}\right) .
\end{aligned}
$$


Thus, by Michael's seletion theorem, we have $h\left(x_{2}\right) \in T x_{2}$ and $z\left(x_{2}\right) \in F x_{2}$. Fix

$$
y_{2} \in \hat{\alpha_{2}} x_{2}+\hat{\beta_{2}}\left(f+x_{2}-N\left(h\left(x_{2}\right), z\left(x_{2}\right)\right)-\lambda A\left(g\left(x_{2}\right)\right)\right)+\hat{\gamma}_{2} v_{2}
$$

Again applying Michael's selection theorem, and letting $w, k: E \rightarrow E$ be continuous mappings such that $w\left(y_{1}\right) \in T\left(y_{1}\right)$ and $k\left(y_{1}\right) \in F\left(y_{1}\right)$, we obtain by Nadler's theorem that there exists $w_{1} \in T\left(y_{2}\right)$ and $k_{1} \in F\left(y_{2}\right)$ such that

$$
\begin{gathered}
\left\|w\left(y_{1}\right)-w_{1}\right\| \leq\left(1+\frac{1}{2}\right) \Theta\left(T\left(y_{1}\right), T\left(y_{2}\right)\right), \\
\left\|k\left(y_{1}\right)-k_{1}\right\| \leq\left(1+\frac{1}{2}\right) \Theta\left(F\left(y_{1}\right), F\left(y_{2}\right)\right) .
\end{gathered}
$$

Choose

$$
x_{3} \in \alpha_{2} x_{2}+\beta_{2}\left(f+y_{2}-N\left(w\left(y_{2}\right), k\left(y_{2}\right)\right)-\lambda A\left(g\left(y_{2}\right)\right)\right)+\gamma_{2} u_{2} .
$$

From $h\left(x_{2}\right) \in T x_{2}, z\left(x_{2}\right) \in F x_{2}$ and Naddler's theorem, there exists $h_{2} \in T x_{3}$ and $z_{2} \in F x_{3}$ such that

$$
\begin{gathered}
\left\|h\left(x_{2}\right)-h_{2}\right\| \leq\left(1+\frac{1}{3}\right) \Theta\left(T x_{2}, T x_{3}\right) \\
\left\|z\left(x_{2}\right)-z_{2}\right\| \leq\left(1+\frac{1}{3}\right) \Theta\left(F x_{2}, F x_{3}\right) .
\end{gathered}
$$

Thus, by Michael's seletion theorem, we have $h\left(x_{3}\right) \in T x_{3}$ and $z\left(x_{3}\right) \in F x_{3}$. Fix

$$
y_{3} \in \hat{\alpha_{3} x_{3}}+\hat{\beta}_{3}\left(f+x_{3}-N\left(h\left(x_{3}\right), z\left(x_{3}\right)\right)-\lambda A\left(g\left(x_{3}\right)\right)\right)+\hat{\gamma}_{3} v_{3}
$$

Applying Michael's selection theorem, and letting $w, k: E \rightarrow E$ be continuous mappings such that $w\left(y_{2}\right) \in T\left(y_{2}\right)$ and $k\left(y_{2}\right) \in F\left(y_{2}\right)$, we obtain by Nadler's theorem that there exists $w_{2} \in$ $T\left(y_{3}\right)$ and $k_{2} \in F\left(y_{3}\right)$ such that

$$
\begin{aligned}
\left\|w\left(y_{2}\right)-w_{2}\right\| & \leq\left(1+\frac{1}{3}\right) \Theta\left(T\left(y_{2}\right), T\left(y_{3}\right)\right), \\
\left\|k\left(y_{2}\right)-k_{2}\right\| & \leq\left(1+\frac{1}{3}\right) \Theta\left(F\left(y_{2}\right), F\left(y_{3}\right)\right) .
\end{aligned}
$$

Choose $x_{4} \in \alpha_{3} x_{3}+\beta_{3}\left(f+y_{3}-N\left(w\left(y_{3}\right), k\left(y_{3}\right)\right)-\lambda A\left(g\left(y_{3}\right)\right)\right)+\gamma_{3} u_{3}$.

Continuing in this way, we obtain the following algorithm.

Algorithm 2.1. Let $h, z, w, k: E \rightarrow E$ be continuous mappings such that for $x_{0} \in E$ arbitary, $h\left(x_{0}\right) \in$ $T x_{0}, z\left(x_{0}\right) \in F\left(x_{0}\right)$. As illustrated above, compute the sequences $\left\{y_{n}\right\},\left\{x_{n}\right\},\left\{h_{n}\right\},\left\{k_{n}\right\},\left\{w_{n}\right\}$, 
and $\left\{z_{n}\right\}$ iteratively such that

(i) $\quad x_{n+1} \in \alpha_{n} x_{n}+\beta_{n}\left(f+y_{n}-N\left(w\left(y_{n}\right), k\left(y_{n}\right)\right)-\lambda A\left(g\left(y_{n}\right)\right)\right)+\gamma_{n} u_{n}$,

(ii) $y_{n} \in \hat{\alpha}_{n} x_{n}+\hat{\beta}_{n}\left(f+x_{n}-N\left(h\left(x_{n}\right), z\left(x_{n}\right)\right)-\lambda A\left(g\left(x_{n}\right)\right)\right)+\hat{\gamma}_{n} v_{n}$,

(iii) $h\left(x_{n}\right) \in T x_{n},\left\|h\left(x_{n}\right)-h_{n}\right\| \leq\left(1+\frac{1}{n+1}\right) \Theta\left(T x_{n}, T x_{n+1}\right)$,

(iv) $z\left(x_{n}\right) \in F x_{n},\left\|z\left(x_{n}\right)-z_{n}\right\| \leq\left(1+\frac{1}{n+1}\right) \Theta\left(F x_{n}, F x_{n+1}\right)$,

(v) $w\left(y_{n}\right) \in T\left(y_{n}\right),\left\|w\left(y_{n}\right)-w_{n}\right\| \leq\left(1+\frac{1}{n+1}\right) \Theta\left(T\left(y_{n}\right), T\left(y_{n+1}\right)\right)$,

(vi) $k\left(y_{n}\right) \in F\left(y_{n}\right),\left\|k\left(y_{n}\right)-k_{n}\right\| \leq\left(1+\frac{1}{n+1}\right) \Theta\left(F\left(y_{n}\right), F\left(y_{n+1}\right)\right), n=0,1,2, \ldots$

The sequence $\left\{x_{n}\right\}$ defined by Algorithm 2.1 is called the Ishikawa-type iterative sequence with bounded errors. In Algorithm 2.1, if $\hat{\beta}_{n}=\hat{\gamma}_{n}=0$ for all $n \geq 0$, then $x_{n}=y_{n}$. Taking $w=h$ and $z=k$, we obtain the following algorithm.

\section{Algorithm 2.2.}

$$
\begin{aligned}
& x_{n+1} \in \alpha_{n} x_{n}+\beta_{n}\left(f+x_{n}-N\left(w\left(y_{n}\right), k\left(y_{n}\right)\right)-\lambda A\left(g\left(x_{n}\right)\right)\right)+\gamma_{n} u_{n}, \\
& h\left(x_{n}\right) \in T\left(x_{n}\right),\left\|h\left(x_{n}\right)-h_{n}\right\| \leq\left(1+\frac{1}{n+1}\right) M\left(T\left(x_{n}\right), T\left(x_{n+1}\right)\right), \\
& z\left(x_{n}\right) \in F\left(x_{n}\right),\left\|z\left(x_{n}\right)-z_{n}\right\| \leq\left(1+\frac{1}{n+1}\right) M\left(F\left(x_{n}\right), F\left(x_{n+1}\right)\right), n=0,1,2, \ldots
\end{aligned}
$$

The sequence $\left\{x_{n}\right\}$ defined by Algorithm 2.2 is called the Mann-type iterative sequence with bounded errors.

\section{Main Results}

3.1. Existence theorem for solutions of set-valued variational inclusion (2.1). In this subsection, we establish an existence theorem for solutions of set-valued variational inclusion (2.1).

Theorem 3.1. Let $E$ be a real uniformly smooth Banach space. Let $T, F: E \rightarrow C B(E)$ and $A: E \rightarrow 2^{E}$ be three set-valued mappings such that $T$ and $F$ are continuous. Let $g: E \rightarrow E$ be a single-valued mapping, and let $N: E \times E \rightarrow E$ be a single-valued continuous mapping. Suppose that the following conditions are satisfied:

(i) $\quad A \circ g: E \longrightarrow 2^{E}$ is $m$ - accretive;

(ii) for $\lambda>0, \lambda A \circ g: E \rightarrow 2^{E}$ is $\mathrm{m}$ - accretive and $\phi$ - expansive;

(iii) the mapping $x \mapsto N(x, y)$ is $\Psi$ - uniformly accretive with respect to the mapping $T$;

(iv) the mapping $y \mapsto N(x, y)$ is accretive with respect to the mapping $F$.

Then, there exist $q \in E, w \in T(q)$, and $k \in F(q)$ such that $(q, w, k)$ is a solution of set-valued variational inclusion (2.1). 
Proof. It follows from conditions (iii) and (iv) and Lemma 2.2 that the mapping $S: E \rightarrow 2^{E}$ defined by $S x=N(T x, F x), \forall x \in E$ is $\Psi$-uniformly accretive. Since $N$ is continuous, and both $T$ and $F$ are continuous, then $S$ is continuous. Hence, $S$ is a continuous and accretive mapping. So, by Morales [30], we have that $S$ is $m$-accretive. From Lemma 2.4(2), $S$ admits a continuous selection $h: E \rightarrow E$, which is $\Psi$-uniformly accretive and $m$-accretive such that $h(x) \in S x=$ $N(T x, F x), \forall x \in E$.

Next, we consider the variational inclusion

$$
f \in h(x)+\lambda A(g(x)),
$$

where $\lambda$ is some positive constant. Since $\lambda A \circ g: E \rightarrow 2^{E}$ is $m$-accretive, $h: E \rightarrow E$ is continuous and $\varphi$-uniformly accretive, then $h+\lambda A \circ g: E \rightarrow 2^{E}$ is $m$-accretive and $\Psi$-uniformly accretive. Moreover, $h+\lambda A \circ g: E \rightarrow 2^{E}$ is also $m$-accretive and $\phi$-expansive. By Lemma 2.6, we have that $h+\lambda A \circ g: E \rightarrow 2^{E}$ is surjective. Therefore, for any given $f \in E$ and $\lambda>0$, there exists a unique $q \in E$ such that $f \in h(q)+\lambda A(g(q))$. The uniqueness of $q \in E$ is a direct consequence of the $\Psi$-uniform accretivity of $h+\lambda A \circ g$. Since $h(q)+\lambda A(g(q)) \subset N(T(q), F(q))+\lambda A(g(q))$, there exist $w \in T(q), k \in F(q)$ such that $f \in N(w, k)+\lambda A(g(q))$. This completes the proof.

Remark 3.1. Theorem 3.1 generalizes and improves of the works of Chang, Kim, and Kim [6, Theorem 3.1]. The Lipschitz continuity assumed on $T$ and $F$ in [6] is replaced with the continuity assumption on $T$ and $F$ in our own case. Moreover, the $\phi$-strongly accretive assumption on the operator $N$ with respect to the mapping $T$ in [6, Theorem 3.1] is extended to the $\Psi$-uniformly accretive assumption on the operator $N$ with respect to the mapping $T$ in our own case.

3.2. Approximate solutions for set-valued variational inclusion (2.1). In Theorem 3.1, we proved that, under mild conditions, there exists unique $q \in E$, and there exist $w \in T(q)$, and $k \in$ $F(q)$, such that $(q, w, k)$ is a solution of set-valued variational inclusion (2.1). In this subsection, we consider solutions of variational inclusion (2.1). In fact, we shall prove the following theorem.

Theorem 3.2. Let $E$ be a real uniformly smooth Banach space. Let $T, F: E \longrightarrow C B(E)$ and $A: E \subset E \longrightarrow 2^{E}$ be three set-valued mappings, let $g: E \longrightarrow E$ be a single-valued mapping, and let $N: E \times E \longrightarrow E$ be a single-valued continuous mapping satisfying the following properties:

(i) $\quad A \circ g: E \longrightarrow 2^{E}$ is $m$-accretive;

(ii) $T, F$ are two continuous mappings and are also $\varphi$-contractive mapping with respect to the Hausdorff metric $\Theta$;

(iii) the mapping $x \mapsto N(x, y)$ is $\Psi$ - uniformly accretive with respect to the mapping $T$;

(iv) the mapping $y \mapsto N(x, y)$ is accretive with respect to the mapping $F$.

Let $\left\{\alpha_{n}\right\},\left\{\beta_{n}\right\},\left\{\gamma_{n}\right\},\left\{\hat{\alpha}_{n}\right\},\left\{\hat{\beta_{n}}\right\}$, and $\left\{\hat{\gamma}_{n}\right\}$ be sequences in $(0,1)$ satisfying the following conditions: (v) $\alpha_{n}+\beta_{n}+\gamma_{n}=\hat{\alpha}_{n}+\hat{\beta}_{n}+\hat{\gamma}_{n}=1$; (vi) $\lim _{n \rightarrow \infty} \beta_{n}=0, \sum_{n=0}^{\infty} \beta_{n}=+\infty$; (vii) $\lim _{n \rightarrow \infty} \hat{\beta}_{n}=$ 0 ; and (viii) $\lim _{n \rightarrow \infty} \hat{\gamma}_{n}=0, \sum_{n=0}^{\infty} \gamma_{n}<\infty$. If ranges $R(I-N(T(),. F())$.$) and R(A \circ g)$ are both bounded, and the sequences $\left\{u_{n}\right\},\left\{v_{n}\right\}$ are bounded sequences in $E$, then the iterative sequences defined by Algorithm 2.1 converge strongly to a solution of set-valued variational inclusion problem (2.1). 
Proof. First, in (i) and (ii) Algorithm 2.1, we choose $d_{n} \in A\left(g\left(x_{n}\right)\right)$ and $e_{n} \in A\left(g\left(y_{n}\right)\right)$ such that

$$
\begin{aligned}
x_{n+1} & =\alpha_{n} x_{n}+\beta_{n}\left(f+y_{n}-N\left(w\left(y_{n}\right), k\left(y_{n}\right)\right)-\lambda e_{n}\right)+\gamma_{n} u_{n}, \\
y_{n} & =\hat{\alpha}_{n} x_{n}+\hat{\beta}_{n}\left(f+x_{n}-N\left(h\left(x_{n}\right), z\left(x_{n}\right)\right)-\lambda d_{n}\right)+\hat{\gamma}_{n} v_{n} .
\end{aligned}
$$

Let

$$
b_{n}:=f+x_{n}-N\left(h\left(x_{n}\right), z\left(x_{n}\right)\right)-\lambda d_{n},
$$

and

$$
c_{n}:=f+y_{n}-N\left(w\left(y_{n}\right), k\left(y_{n}\right)\right)-\lambda e_{n} .
$$

Then, (3.2) can be written as

$$
\begin{aligned}
x_{n+1} & =\alpha_{n} x_{n}+\beta_{n} c_{n}+\gamma_{n} u_{n}, \\
y_{n} & =\hat{\alpha}_{n} x_{n}+\hat{\beta}_{n} b_{n}+\hat{\gamma}_{n} v_{n}, \quad n \geq 0 .
\end{aligned}
$$

Since $\left\{u_{n}\right\}$ and $\left\{v_{n}\right\}$ are bounded sequences and ranges of $R(I-N(T(),. F())$.$) and R(A \circ g)$ are bounded subsets of $E$, then, for the unique $q \in E$, and for some $w \in T(q)$, and $k \in F(q)$ such that $(q, w, k)$ is a solution of the set-valued variational inclusion (2.1), we let $d$ be given by

$$
d=\max \left\{\sup _{n \geq 0}\left\{\left\|u_{n}-q\right\|\right\}, \sup _{n \geq 0}\left\{\left\|v_{n}-q\right\|\right\}, \sup _{w \in \Omega_{x}}\{\|w-q\|\},\left\|x_{1}-q\right\|,\left\|x_{0}-q\right\|\right\}
$$

where $\Omega_{x}=\{u: u \in f+x-N(T(x), F(x))-\lambda A(g(x))\}, \forall x \in E$. Thus,

$$
\begin{aligned}
& \left\|c_{n}-q\right\| \leq d, \text { for all } \mathrm{n} \geq 0, \\
& \left\|b_{n}-q\right\| \leq d, \text { for all } \mathrm{n} \geq 0 .
\end{aligned}
$$

Next, we prove that

$$
\left\|x_{n}-q\right\| \leq d \text { and }\left\|y_{n}-q\right\| \leq d, \text { for all } \mathrm{n} \geq 0 .
$$

In fact, by the defintion of $d$, we know that $\left\|x_{0}-q\right\| \leq d$ and $\left\|x_{1}-q\right\| \leq d$. Again by (3.3), we have that

$$
\begin{aligned}
\left\|x_{2}-q\right\| & =\left\|\alpha_{1}\left(x_{1}-q\right)+\beta_{1}\left(c_{1}-q\right)+\gamma_{1}\left(u_{1}-q\right)\right\| \\
& \leq \alpha_{1} d+\beta_{1} d+\gamma_{1} d \\
& =d .
\end{aligned}
$$

By induction, we can prove that $\left\|x_{n}-q\right\| \leq d$. To see this, we observe from above that $\left\|x_{0}-q\right\| \leq$ $d$. Assume that it is true for some $n=k \geq 0$, that is, $\left\|x_{k}-q\right\| \leq d$. We show that it is true for $n=k+1$. Now,

$$
\begin{aligned}
\left\|x_{k+1}-q\right\| & =\left\|\alpha_{k}\left(x_{k}-q\right)+\beta_{k}\left(c_{k}-q\right)+\gamma_{k}\left(u_{k}-q\right)\right\| \\
& \leq \alpha_{k} d+\beta_{k} d+\gamma_{k} d \\
& =d .
\end{aligned}
$$


Therefore, we have that $\left\|x_{n}-q\right\| \leq d, \forall n \geq 0$. Moreover, we also have

$$
\begin{aligned}
\left\|y_{n}-q\right\| & =\left\|\hat{\alpha}_{n}\left(x_{n}-q\right)+\hat{\beta}_{n}\left(b_{n}-q\right)+\hat{\gamma}_{n}\left(v_{n}-q\right)\right\| \\
& \leq \hat{\alpha_{n}}\left\|x_{n}-q\right\|+\hat{\beta}_{n}\left\|b_{n}-q\right\|+\hat{\gamma}_{n}\left\|v_{n}-q\right\| \\
& \leq d .
\end{aligned}
$$

So, (3.5) is proved.

Next, using (3.3)-(3.5) and Lemma 2.1, we obtain that

$$
\begin{aligned}
\left\|x_{n+1}-q\right\|^{2} \leq & \alpha_{n}^{2}\left\|x_{n}-q\right\|^{2}+2 \beta_{n}\left\langle c_{n}-q, j\left(y_{n}-q\right)\right\rangle \\
& +2 \beta_{n}\left\langle c_{n}-q, j\left(x_{n+1}-q\right)-j\left(y_{n}-q\right)\right\rangle \\
& +2 \gamma_{n}\left\langle u_{n}-q, j\left(x_{n+1}-q\right)\right\rangle .
\end{aligned}
$$

Observe that

$$
\begin{aligned}
& \left\|x_{n+1}-q-\left(y_{n}-q\right)\right\| \\
\leq \quad & \alpha_{n}\left\|x_{n}-y_{n}\right\|+\beta_{n}\left\|c_{n}-y_{n}\right\|+\gamma_{n}\left\|u_{n}-y_{n}\right\| \\
\leq \quad & \alpha_{n}\left\|x_{n}-\left(\hat{\alpha_{n}} x_{n}+\hat{\beta}_{n} b_{n}+\hat{\gamma}_{n} v_{n}\right)\right\|+\beta_{n}\left(\left\|c_{n}-q\right\|+\left\|y_{n}-q\right\|\right) \\
& +\gamma_{n}\left(\left\|u_{n}-q\right\|+\left\|y_{n}-q\right\|\right) \\
\leq & \alpha_{n}\left[\hat{\beta_{n}}\left\|x_{n}-b_{n}\right\|+\hat{\gamma}_{n}\left\|x_{n}-v_{n}\right\|\right]+\beta_{n}\left\|c_{n}-q\right\|+\gamma_{n}\left\|u_{n}-q\right\| \\
& +\left(\beta_{n}+\gamma_{n}\right)\left\|y_{n}-q\right\| \\
\leq & \alpha_{n} \hat{\beta_{n}}\left\|x_{n}-q\right\|+\alpha_{n} \hat{\beta_{n}}\left\|b_{n}-q\right\|+\alpha_{n} \hat{\gamma}_{n}\left\|x_{n}-q\right\|+\alpha_{n} \hat{\gamma}_{n}\left\|v_{n}-q\right\| \\
& +\beta_{n}\left\|c_{n}-q\right\|+\gamma_{n}\left\|u_{n}-q\right\|+\left(\beta_{n}+\gamma_{n}\right)\left\|y_{n}-q\right\| \\
= & \left(\alpha_{n} \hat{\beta_{n}}+\alpha_{n} \hat{\gamma_{n}}\right)\left\|x_{n}-q\right\|+\alpha_{n} \hat{\beta}_{n}\left\|b_{n}-q\right\|+\alpha_{n} \hat{\gamma_{n}}\left\|v_{n}-q\right\| \\
& +\beta_{n}\left\|c_{n}-q\right\|+\gamma_{n}\left\|u_{n}-q\right\|+\left(\beta_{n}+\gamma_{n}\right)\left\|y_{n}-q\right\| \\
\leq & \alpha_{n}\left(\hat{\beta_{n}}+\hat{\gamma_{n}}\right) d+\alpha_{n} \hat{\beta_{n}} d+\alpha_{n} \hat{\gamma}_{n} d+\beta_{n} d+\gamma_{n} d+\left(\beta_{n}+\gamma_{n}\right) d .
\end{aligned}
$$

Thus, $\lim _{n \rightarrow \infty}\left\|x_{n+1}-q-\left(y_{n}-q\right)\right\|=0$. Since $j$ is uniformly continuous on any bounded subsets of $E$, we have that $\lim _{n \rightarrow \infty}\left\|j\left(x_{n+1}-q\right)-j\left(y_{n}-q\right)\right\|=0$. Setting

$$
\xi_{n}=\left|\left\langle c_{n}-q, j\left(x_{n+1}-q\right)-j\left(y_{n}-q\right)\right\rangle\right|,
$$

we obtain that $\lim _{n \rightarrow \infty} \xi_{n}=0$ due to

$$
\begin{aligned}
\zeta_{n} & =\left|\left\langle c_{n}-q, j\left(x_{n+1}-q\right)-j\left(y_{n}-q\right)\right\rangle\right| \\
& \leq\left\|c_{n}-q\right\|\left\|j\left(x_{n+1}-q\right)-j\left(y_{n}-q\right)\right\| \\
& \leq d\left\|j\left(x_{n+1}-q\right)-j\left(y_{n}-q\right)\right\| \rightarrow 0 \text { as } n \rightarrow \infty .
\end{aligned}
$$

Next, since $w\left(y_{n}\right) \in T\left(y_{n}\right), k\left(y_{n}\right) \in F\left(y_{n}\right)$, and $e_{n} \in A g\left(y_{n}\right)$, one has

$$
N\left(w\left(y_{n}\right), k\left(y_{n}\right)\right)+\lambda e_{n} \in N\left(T\left(y_{n}\right), F\left(y_{n}\right)\right)+\lambda A g\left(y_{n}\right) .
$$

Since $q$ is the unique solution of the variational inclusion problem, one has $f \in h(q)+\lambda A g(q)$. This shows that $f \in N(T(q), F(q))+\lambda A g(q)$. By the assumptions of Theorem 3.2, the mapping 
$N(T(),. F())+.\lambda A g():. E \longrightarrow 2^{E}$ is $\Psi$-uniformly accretive. Thus,

$$
\begin{aligned}
\left\langle f-\left(N\left(w\left(y_{n}\right), k\left(y_{n}\right)\right)+\lambda e_{n}\right), j\left(y_{n}-q\right)\right\rangle & =-\left\langle N\left(w\left(y_{n}\right), k\left(y_{n}\right)\right)+\lambda e_{n}-f, j\left(y_{n}-q\right)\right\rangle \\
& \leq-\varphi\left(\left\|y_{n}-q\right\|\right) .
\end{aligned}
$$

It follows that

$$
\begin{aligned}
& 2 \beta_{n}\left\langle c_{n}-q, j\left(y_{n}-q\right)\right\rangle \\
= & 2 \beta_{n}\left\langle f+y_{n}-N\left(w\left(y_{n}\right), k\left(y_{n}\right)\right)-\lambda e_{n}-q, j\left(y_{n}-q\right)\right\rangle \\
= & 2 \beta_{n}\left[\left\langle y_{n}-q, j\left(y_{n}-q\right)\right\rangle-\left\langle N\left(w\left(y_{n}\right), k\left(y_{n}\right)\right)+\lambda e_{n}-f, j\left(y_{n}-q\right)\right\rangle\right] \\
\leq & 2 \beta_{n}\left[\left\|y_{n}-q\right\|^{2}-\Psi\left(\left\|y_{n}-q\right\|\right)\right] .
\end{aligned}
$$

Substituting (3.9) and (3.8) into (3.6), we get

$$
\left\|x_{n+1}-q\right\|^{2} \leq \alpha_{n}^{2}\left\|x_{n}-q\right\|^{2}+2 \beta_{n}\left[\left\|y_{n}-q\right\|^{2}-\Psi\left(\left\|y_{n}-q\right\|\right)\right]+2 \beta_{n} \zeta_{n}+2 \gamma_{n} d^{2} .
$$

Furthermore, from (3.3), (3.5) and Lemma 2.1, we observe that

$$
\begin{aligned}
\left\|y_{n}-q\right\|^{2} & \leq \hat{\alpha_{n}}\left\|x_{n}-q\right\|^{2}+2 \hat{\beta}_{n}\left\langle b_{n}-q, j\left(y_{n}-q\right)\right\rangle+2 \hat{\gamma}_{n}\left\langle v_{n}-q, j\left(y_{n}-q\right)\right\rangle \\
& \leq\left\|x_{n}-q\right\|^{2}+2 \hat{\beta_{n}}\left\|b_{n}-q\right\|\left\|y_{n}-q\right\|+2 \hat{\gamma}_{n}\left\|v_{n}-q\right\|\left\|y_{n}-q\right\| \\
& \leq\left\|x_{n}-q\right\|^{2}+2 d^{2}\left(\hat{\beta}_{n}+\hat{\gamma}_{n}\right)
\end{aligned}
$$

Thus, it follows from (3.11) that

$$
\left\|y_{n}-q\right\|^{2} \leq\left\|x_{n}-q\right\|^{2}+2 d^{2}\left(\hat{\beta}_{n}+\hat{\gamma}_{n}\right) .
$$

Observe that $2 d^{2}\left(\hat{\beta}_{n}+\hat{\gamma}_{n}\right) \rightarrow \infty$ as $n \rightarrow \infty$. Substituting (3.12) into (3.10), we have that

$$
\begin{aligned}
& \left\|x_{n+1}-q\right\|^{2} \\
\leq & \alpha_{n}^{2}\left\|x_{n}-q\right\|^{2}+2 \beta_{n}\left[\left\|y_{n}-q\right\|^{2}-\Psi\left(\left\|y_{n}-q\right\|\right)\right]+2 \beta_{n} \zeta_{n}+2 d^{2} \gamma_{n} \\
\leq & \alpha_{n}^{2}\left\|x_{n}-q\right\|^{2}+2 \beta_{n}\left[\left\|x_{n}-q\right\|^{2}+2 d^{2}\left(\hat{\beta}_{n}+\hat{\gamma}_{n}\right)\right]-2 \beta_{n} \Psi\left(\left\|y_{n}-q\right\|\right)+2 \beta_{n} \zeta_{n}+2 d^{2} \gamma_{n} \\
= & \left(1-\left(\beta_{n}+\gamma_{n}\right)\right)^{2}\left\|x_{n}-q\right\|^{2}+2 \beta_{n}\left\|x_{n}-q\right\|^{2}+4 d^{2} \beta_{n}\left(\hat{\beta}_{n}+\hat{\gamma}_{n}\right) \\
& -2 \beta_{n} \Psi\left(\left\|y_{n}-q\right\|\right)+2 \beta_{n} \zeta_{n}+2 d^{2} \gamma_{n} \\
= & \left\|x_{n}-q\right\|^{2}-2 \beta_{n}\left\|x_{n}-q\right\|^{2}-2 \gamma_{n}\left\|x_{n}-q\right\|^{2}+\left(\beta_{n}+\gamma_{n}\right)^{2}\left\|x_{n}-q\right\|^{2} \\
& +2 \beta_{n}\left\|x_{n}-q\right\|^{2}+4 \beta_{n} d^{2}\left(\hat{\beta}_{n}+\hat{\gamma}_{n}\right)-2 \beta_{n} \Psi\left(\left\|y_{n}-q\right\|\right)+2 \beta_{n} \zeta_{n}+2 d^{2} \gamma_{n} \\
\leq & \left\|x_{n}-q\right\|^{2}+\left[-2 \gamma_{n}+2 \gamma_{n}+\beta_{n}^{2}+\gamma_{n}^{2}\right]\left\|x_{n}-q\right\|^{2}+4 d^{2} \beta_{n}\left(\hat{\beta}_{n}+\hat{\gamma}_{n}\right) \\
& -2 \beta_{n} \Psi\left(\left\|y_{n}-q\right\|\right)+2 \beta_{n} \zeta_{n}+2 d^{2} \gamma_{n} \\
\leq & \left\|x_{n}-q\right\|^{2}+d^{2}\left(\beta_{n}^{2}+\gamma_{n}^{2}\right)+4 d^{2} \beta_{n}\left(\hat{\beta_{n}}+\hat{\gamma}_{n}\right)-2 \beta_{n} \Psi\left(\left\|y_{n}-q\right\|\right)+2 \beta_{n} \zeta_{n}+2 d^{2} \gamma_{n} \\
\leq & \left\|x_{n}-q\right\|^{2}-\beta_{n} \Psi\left(\left\|y_{n}-q\right\|\right)-\beta_{n}\left[\Psi\left(\left\|y_{n}-q\right\|\right)-2 \mu_{n}-d^{2} \beta_{n}-2 \zeta_{n}\right]+3 d^{2} \gamma_{n}
\end{aligned}
$$

Let $\sigma=\inf _{n \geq 0}\left\|y_{n}-q\right\|$, Next, we prove that $\sigma=0$. Suppose for contradiction that $\sigma>0$. Then $\left\|y_{n}-q\right\| \geq \sigma>0, \forall n \geq 0$. By the strictly increasing property of $\varphi$ with $\varphi(0)=0$, we have $\varphi(\sigma)>0$. Hence, it follows from (3.13) that

$$
\left\|x_{n+1}-q\right\|^{2} \leq\left\|x_{n}-q\right\|^{2}-\beta_{n} \Psi(\sigma)-\beta_{n}\left[\Psi(\sigma)-2 \mu_{n}-\beta_{n} d^{2}-2 \zeta_{n}\right]+3 \gamma_{n} d^{2} .
$$


Since $\beta_{n} \rightarrow 0, \mu_{n} \rightarrow 0$ and $\zeta_{n} \rightarrow 0$, there exists $n_{0}$ such that, for all $n \geq n_{0}, \varphi(\sigma)-2 \mu_{n}-$ $\beta_{n} d^{2}-2 \zeta_{n}>0$. Therefore, for $n \geq n_{0}$, we have

$$
\left\|x_{n+1}-q\right\|^{2} \leq\left\|x_{n}-q\right\|^{2}-\beta_{n} \Psi(\sigma)+3 d^{2} \gamma_{n}
$$

that is, $\beta_{n} \Psi(\sigma) \leq\left\|x_{n}-q\right\|^{2}-\left\|x_{n+1}-q\right\|^{2}+3 d^{2} \gamma_{n}, \forall n \geq n_{0}$. Hence, for any $m \geq n_{0}$, we have

$$
\begin{aligned}
\Psi(\sigma) \sum_{n=n_{0}}^{m} \beta_{n} & \leq\left\|x_{n_{0}}-q\right\|^{2}-\left\|x_{m+1}-q\right\|^{2}+3 d^{2} \sum_{n=n_{0}}^{m} \gamma_{n} \\
& \leq\left\|x_{n_{0}}-q\right\|^{2}+3 d^{2} \sum_{n=n_{0}}^{m} \gamma_{n} .
\end{aligned}
$$

Letting $m \rightarrow \infty$ yields that $+\infty \leq\left\|x_{n_{0}}-q\right\|^{2}+3 d^{2} \sum_{n=n_{0}}^{\infty} \gamma_{n}<+\infty$, which is a contradiction. Hence, we have that $\sigma=0$. Therefore, there exists a subsequence $\left\{y_{n_{j}}\right\} \subseteq\left\{y_{n}\right\}$ such that

$$
y_{n_{j}} \rightarrow \text { as } j \rightarrow \infty
$$

By (3.7) and (3.15), we see that

$$
\left\|x_{n_{j}+1}-q\right\| \leq\left\|x_{n_{j}+1}-q-\left(y_{n_{j}}-q\right)\right\|+\left\|y_{n_{j}}-q\right\|
$$

Thus, $\lim _{j \rightarrow \infty}\left\|x_{n_{j}+1}-q\right\|=0$. That is,

$$
x_{n_{j}+1}=\alpha_{n_{j}} x_{n_{j}}+\beta_{n_{j}} c_{n_{j}}+\gamma_{n_{j}} \rightarrow q \text { as } j \rightarrow \infty
$$

Since $\beta_{n} \rightarrow 0, \gamma_{n} \rightarrow 0, \hat{\beta_{n}} \rightarrow 0,\left\{c_{n}\right\},\left\{b_{n}\right\},\left\{u_{n}\right\}$ and $\left\{v_{n}\right\}$ are bounded sequences, this implies that $x_{n_{j}} \rightarrow q$ as $j \rightarrow \infty$. Therefore,

$$
y_{n_{j}}=\hat{\alpha_{n_{j}}} x_{n_{j}}+\hat{\beta_{n_{j}}} b_{n_{j}}+\hat{\gamma_{n_{j}}} v_{n_{j}} \rightarrow q \text { as } j \rightarrow \infty \text {. }
$$

We can easily verify by induction that, for all $i \geq 0, x_{n_{j}+i} \rightarrow q$ and $y_{n_{j}+i} \rightarrow q$ as $j \rightarrow \infty$. Therefore, $x_{n} \rightarrow q$ and $y_{n} \rightarrow q$ as $n \rightarrow \infty$. Thus, by the continuity of $h, z, w$ and $k, h\left(x_{n}\right) \rightarrow h(q), z\left(x_{n}\right) \rightarrow$ $z(q), w\left(y_{n}\right) \rightarrow w(q)$ and $k\left(y_{n}\right) \rightarrow k(q)$. Furthermore, since $\Theta(T x, T y) \leq \varphi(\|x-y\|)$, it follows from the fact that $\varphi$ is continuous that

$$
\begin{aligned}
\left\|h\left(x_{n}\right)-h_{n}\right\| & \leq\left(1+\frac{1}{n}\right) \Theta\left(T x_{n}, T x_{n+1}\right) \\
& \leq\left(1+\frac{1}{n}\right) \varphi\left(\left\|x_{n}-x_{n+1}\right\|\right) \rightarrow 0 \text { as } n \rightarrow \infty, \text { and } \\
\left\|z\left(x_{n}\right)-z_{n}\right\| & \leq\left(1+\frac{1}{n}\right) \Theta\left(F x_{n}, F x_{n+1}\right) \\
& \leq\left(1+\frac{1}{n}\right) \varphi\left(\left\|x_{n}-x_{n+1}\right\|\right) \rightarrow 0 \text { as } n \rightarrow \infty .
\end{aligned}
$$


This implies by the continuity of $h$ and $z$ that $\left\{h_{n}\right\}$ and $\left\{z_{n}\right\}$ converge to $h(q)=h^{*} \in T(q)$ and $z(q)=$ $z^{*} \in F(q)$, respectively. Furthermore, we also have

$$
\begin{aligned}
\left\|w\left(y_{n}\right)-w_{n}\right\| & \leq\left(1+\frac{1}{n}\right) \Theta\left(T\left(y_{n}\right), T\left(y_{n+1}\right)\right) \\
& \leq\left(1+\frac{1}{n}\right) \varphi\left(\left\|y_{n}-y_{n+1}\right\|\right) \\
& \leq\left(1+\frac{1}{n}\right) \varphi\left(\left\|y_{n}-y_{n+1}\right\|\right) \rightarrow 0 \text { as } n \rightarrow \infty \text { and } \\
\left\|k\left(y_{n}\right)-k_{n}\right\| & \leq\left(1+\frac{1}{n}\right) \Theta\left(F\left(y_{n}\right), F\left(y_{n+1}\right)\right) \\
& \leq\left(1+\frac{1}{n}\right) \varphi\left(\left\|y_{n}-y_{n+1}\right\|\right) \rightarrow 0 \text { as } n \rightarrow \infty .
\end{aligned}
$$

From the continuity of $w$ and $k$, we have that $\left\{w_{n}\right\}$ and $\left\{k_{n}\right\}$ converge to $w(q)=w^{*} \in T(q)$ and $k(q)=k^{*} \in F(q)$, respectively.

Next, we prove that $h^{*}=w^{*}=w$ and $z^{*}=k^{*}=k$, where $(q, w, k), q \in E, w \in T(q)$, and $k \in$ $F(q)$ is the solution of the set-valued variational inclusion. In fact, since

$$
\begin{aligned}
d\left(w^{*}, T(q)\right) & \leq d\left(w^{*}, w_{n}\right)+d\left(w_{n}, T(q)\right) \\
& \leq d\left(w^{*}, w_{n}\right)+\Theta\left(T\left(y_{n}\right), T(q)\right) \\
& \leq d\left(w^{*}, w_{n}\right)+\varphi\left(\left\|y_{n}-q\right\|\right) \rightarrow 0 \text { as } n \rightarrow \infty,
\end{aligned}
$$

which implies that $w^{*} \in T(q)$. Similarly, we can prove that $h^{*} \in T(q)$. Observe that

$$
\begin{aligned}
d\left(w^{*}, w\right) & \leq d\left(w^{*}, w_{n}\right)+d\left(w_{n}, w\right) \\
& \leq d\left(w^{*}, w_{n}\right)+\Theta\left(T\left(y_{n}\right), T(q)\right) \\
& \leq d\left(w^{*}, w_{n}\right)+\varphi\left(\left\|y_{n}-q\right\|\right) \rightarrow 0 \text { as } n \rightarrow \infty
\end{aligned}
$$

This implies that $w^{*}=w$. In view of

$$
\begin{aligned}
d\left(h^{*}, w\right) & \leq d\left(h^{*}, h_{n}\right)+d\left(h_{n}, w\right) \\
& \leq d\left(h^{*}, h_{n}\right)+M\left(T x_{n}, T q\right) \\
& \leq d\left(h^{*}, h_{n}\right)+\varphi\left(\left\|x_{n}-q\right\|\right) \rightarrow 0 \text { as } n \rightarrow \infty,
\end{aligned}
$$

we obtain that $h^{*}=w$. Following the same argument, we can prove that $z^{*} \in F(q), k^{*} \in F(q)$, and $z^{*}=k^{*}=k$. Consequently, we have that the sequences $\left\{x_{n}\right\},\left\{w_{n}\right\}$, and $\left\{k_{n}\right\}$ defined by (2.5) respectively converge strongly to $q, w$ and $k$, which satisfy the set-valued variational inclusion. This completes the prove.

Remark 3.2. All the observations in Section 1 are properly addressed. It also deserves mentioning that we can also easily solve set-valued variational inclusions 1.1 via Algorithm 2.2 following the prof of Theorem 3.2. Our theorems extend, generalize, improve, and unify the corresponding results of $[5,6,7,8,10,16,11,12,13,14,15,17,18,19,20]$.

\section{Acknowledgments}


The authors would like to thank the Simons Foundation and the coordinators of the Simons Foundation for Sub-Sahara Africa Nationals with base at Department of Mathematics and Statistical Sciences, Botswana International University of Science and Technology, Botswana, for providing financial support that helped in carrying out this research. We are also thankful to the anonymous referees whose suggestions helped to improve the quality of this paper.

\section{REFERENCES}

[1] Y. Censor, S. Petra, C. Schnorr, Superiorization vs. accelerated convex optimization: The superiorized / regularized least-squares case, J. Appl. Numer. Optim. 2 (2020), 15-62.

[2] X. Qin, N.T. An, Smoothing algorithms for computing the projection onto a Minkowski sum of convex sets, Comput. Optim. Appl. 74 (2019), 821-850.

[3] E. Bonacker, A. Gibali, K.H. Kufer, Nesterov perturbations and projection methods applied to IMRT, J. Nonlinear Var. Anal. 4 (2020), 63-86.

[4] T.H. Cuong, J.C. Yao, N.D. Yen, Qualitative properties of the minimum sum-of-squares clustering problem, Optimization, 69 (2020), 2131-2154.

[5] M. A. Noor, Generalized set-valued variational inclusions and resolvent equations, J. Math. Anal. Appl. 228 (1998), 206-220.

[6] S. S. Chang, J. K. Kim, K. H. Kim, On the existence and iterative approximation problems of solutions for set-valued variational inclusions in Banach spaces, J. Math. Anal. Appl. 268 (2002), 89-108.

[7] S. S. Chang, Some problems and results in the study of nonlinear analysis, Nonlinear Anal. 30 (1997), 41974208.

[8] S. S. Chang, The Mann and Ishikawa iterative approximation of solutions to variational inclusions with accretive type mappings, Comput. Math. Appl. 37 (1999), 17-24.

[9] C.E. Chidume, Geometric Properties of Banach Spaces and Nonlinear Iterations, Lecture Notes in Mathematics, Vol. 1965, p. 326, 2009.

[10] X. P. Ding, Iterative process with errors of nonlinear equations involving m-accretive operators, J. Math. Anal. Appl. 209 (1997), 191-201.

[11] M. A. Noor, K. I. Noor, Th. M. Rassias, Set-valued resolvent equations and mixed variational inequalities, J. Math. Anal. Appl. 220 (1998), 741-759.

[12] M. A. Noor, Algorithms for general monotone mixed variational inequalities, J. Math. Anal. Appl. 229 (1999), 330-343.

[13] M. A. Noor, Numerical methods for monotone mixed variational inequalities, Adv. Nonlinear Var. Inequal. 1 1998, 51-79.

[14] M. A. Noor, An in implicit method for mixed variational inequalities, Appl. Math. Lett. 11 (1998), 109-113.

[15] M. A. Noor, Generalized multi-valued quasi-variational inequalities (II), Comput. Math. Appl. 355 (1997), 63-78.

[16] N.J. Huang, On the generalized implicit quasi-variational inequalities, J. Math. Anal. Appl. 216 (1997), 197210.

[17] J. S. Jung, C. H. Morales, The Mann process for perturbed m-accretive operators in Banach spaces, Nonlinear Anal. 46 (2001), 231-243.

[18] K. R. Kazmi, Mann and Ishikawa type perturbed iterative algorithms for generalized quasi-varaitional inclusions, J. Math. Anal. Appl. 209 (1997), 572-584.

[19] X. Ding, Salahuddin, A system of general nonlinear variational inclusions in Banach spaces, Appl. Math. Mech. 36 (2015) 1663-1672.

[20] A. Hassouni, A. Moudafi, A perturbed algorithm for variational inclusions, J. Math. Anal. Appl. 185 (1994), 706-712.

[21] Y. Alber, R. Espinola, P. Lorenzo, Strongly Convergent Approximations to fixed points of total asymptotically nonexpansive mappings, Acta Math. Sinica, 24 (2008), 1005-1022.

[22] E. U. Ofoedu, Strong convergence theorem for uniformly L-Lipschitzian asymptoitcally pseudocontractive mapping in real Banach space, J. Math. Anal. Appl. 321 (2006), 722-728. 
[23] L. S. Liu, Ishikawa and Mann itrative process with errors for nonlinear strongly accretive mappings in Banach spaces, J. Math. Anal. Appl. 45 (1995), 114-125.

[24] Y. Xu, Ishikawa and Mann iterative process with errors for nonlinear strongly accretive operator equations, J. Math. Anal. Appl. 224 (1998), 98-101.

[25] K. Deimling, Nonlinear Functional Analysis, Springer-Verlag, Berlin, 1985.

[26] S. B. Nadler, Multi-valued contraction mappings, Pacific J. Math. 30 (1969), 475-488.

[27] R. E. Bruck, Jr., S. Reich, Accretive operators, Banach limits and dual ergodic theorems, Bull. Acad. Polon. Sci. 12 (1981), 585-589.

[28] E. Michael, Continuous solutions I, Ann. Math. 63 (1956), 361-382.

[29] R. H. Martin, Jr., A global existence theorem for autonomous differential equations in Banach spaces, Proc. Amer. Math. Soc. 26 (1970), 307-314.

[30] C. Morales, Surjectivity theorem for multi-valued mappings of accretive type, Comment. Math. Univ. Carolin. 26 (1985), 397-413. 